\title{
A Stochastic Model for File Lifetime and Security in Data Center Networks ${ }^{\star}$
}

\author{
Quan-Lin Li, Fan-Qi Ma, and Jing-Yu Ma \\ School of Economics and Management Sciences, Yanshan University, Qinhuangdao \\ 066004, China \\ liquanlin@tsinghua.edu.cn
}

\begin{abstract}
Data center networks are an important infrastructure in various applications of modern information technologies. Note that each data center always has a finite lifetime, thus once a data center fails, then it will lose all its storage files and useful information. For this, it is necessary to replicate and copy each important file into other data centers such that this file can increase its lifetime of staying in a data center network. In this paper, we describe a large-scale data center network with a file $d$-threshold policy, which is to replicate each important file into at most $d-1$ other data centers such that this file can maintain in the data center network under a given level of data security in the long-term. To this end, we develop three relevant Markov processes to propose two effective methods for assessing the file lifetime and data security. By using the $R G$-factorizations, we show that the two methods are used to be able to more effectively evaluate the file lifetime of large-scale data center networks. We hope the methodology and results given in this paper are applicable in the file lifetime study of more general data center networks with replication mechanism.
\end{abstract}

Keywords: Data center · replication mechanism · file lifetime · data security $\cdot$ Markov process $\cdot R G$-factorization.

\section{Introduction}

Data center networks are an important infrastructure for various applications of modern information technologies. However, not only does each data center have a finite lifetime, but it is also possible to suffer from natural disasters and man-made damages. Once a data center fails, then it will lose all its storage files and useful information. In practice, such a data center failure has caused key innovation in design and management of large-scale data centers, one of which is how to keep that no important file is lost in the data center network in the long-term. To do this, it is necessary to increase file and data availability as far as possible. In this case, a file copy redundancy technology is developed as a

\footnotetext{
* Quan-Lin Li was supported by the National Natural Science Foundation of China under grant No. 71671158 and No. 71471160, and by the Natural Science Foundation of Hebei province under grant No. G2017203277.
} 
simple mode that each important file is replicate into a subset of data centers according to a comprehensive consideration for departments and/or geographical environment, together with emergency responses for natural disasters and manmade damages. Therefore, during the last decade considerable attention has been paid to developing stochastic model analysis for assessing file lifetime and data security in large-scale data center networks with file replication mechanism. Also see Picconi et al. [11] and [12, Kersch and Szabo [5] and Feuillet and Robert [4] for more details.

Little work has been done on how to establish stochastic models (e.g., Markov processes, queueing theory and stochastic game) to assess the file lifetime and data security in a large-scale data center network with file replication mechanism. Intuitively, such a study is more interesting, difficult and challenging due to the fact that the mathematical modelling is based on reliability and security analysis of large-scale stochastic networks. Based on this, important topics include failure prediction for data centers, how to control new data centers joining this network, how to design and optimize file replication mechanism. In addition, there are still some interesting issues, such as cost analysis of recovering lost data, effect of file replication mechanism and bandwidth limitation, durability and availability of data, and how to control the file lost probability. Readers may refer to recent publications for details, among which, data storage systems by Blake and Rodrigues [2, Utard and Vernois [18, Lian et al. [7, Chun [3] and Ramabhadran and Pasquale [14, [15] and [16]; DHT replication by Picconi et al. 11] and [12, Kersch and Szabo [5], Pace et al. [10] and Kniesburges et al. [6]; failure prediction by Pinheiro et al. [13]; and large-scale stochastic networks with unreliable processors by Feuillet and Robert [4, Sun et al. [17] and Aghajani et al. [1].

The main contributions of this paper are twofold. The first one is to describe a large-scale data center network, in which each data center may fail and new data centers can join this network, and a file $d$-replication policy is proposed to increase file lifetime and data security. The second one is to develop three relevant Markov processes, which lead to two effective methods for assessing the file lifetime and data security in the data center network. By using the $R G$ factorizations of any absorbing Markov process, we show that the two methods can be very effective in file lifetime analysis of more general data center networks with file replication mechanism. Finally, we use numerical examples to indicate impact of the threshold $d$ on the file average lifetime in this data center network.

The remainder of this paper is organized as follows. Section 2 describes the data center network with file replication mechanism. Section 3 develops two relevant Markov processes to give an approximate method for assessing file average lifetime. Section 4 establishes a QBD process to propse a two-dimensional method for assessing file average lifetime, and uses a numerical example to indicate impact of the threshold $d$ on the file average lifetime in this data center network. 


\section{Model Description}

In this section, we describe a large-scale data center network with file replication mechanism, in which each data center may fail and new data centers can join this network, and a file $d$-replication policy is proposed to increase the file lifetime and data security in the data center network.

For a large-scale data center network, we describe its physical structure, main random factors and system parameters as follows:

(1) The physical structure: There are many data centers distributed in parallel a physical network with different departments and/or geographical environment. For simplicity of analysis, we assume that all the data centers are identical and are operated independently.

(2) The lifetime: Each data center in this network may be failure. We assume that the lifetime $X$ of each data center follows an exponential distribution with failure rate $\lambda>0$, that is, $P\{X \leq t\}=1-e^{-\lambda t}$. Obviously, $E[X]=1 / \lambda$. If there are $k$ data centers in this network, then the failure rate of the data center network is $k \lambda$ due to the exponential lifetime of each data center.

(3) A joining process of new data centers: Since the data center networks not only are fast developing in the last over ten years but also each data center may be failure, new data centers need to continually join to the network such that the data center network can maintain a development of sustainability through many incessant equipment replacements. We assume that the inputs of new data centers to the data centers network is a poisson process with arrival rate $\beta>0$.

(4) A file $d$-replication policy: We assume that each file is stored in at most $d \geq 1$ data centers in this network. Once the copy number of the file is less than $d$ and there also exists an available data center without storing the file, then the file will fast replicated to the data center. We assume that the copy time $Y$ of the file replicated to the available data center follows an exponential distribution with copy rate $\mu>0$, that is, $P\{Y \leq t\}=1-e^{-\mu t}$. Obviously, $E[Y]=1 / \mu$. If there are $k$ identical copy files be being duplicated to $k$ different data centers, then the copy time distribution of the $k$ identical copy files is exponential with copy rate $k \mu$.

(5) The file lost process: Once a data center fails, then all its files and useful information in the data center will be lost immediately.

We assume that all the random variables involved in the data center network are independent of each other.

\section{An Approximate Assessment Method of File Lifetime}

In this section, we first set up a birth-death process to study the steady-state probability distribution of the number of available data centers in the data center network. Then we establish another birth-death process to give an approximate assessment method of file lifetime in the the data center network with file $d$ replication policy. 


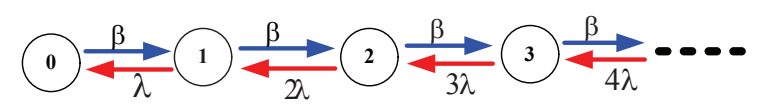

Fig. 1. State transition relation of a birth-death process

\subsection{The number of available data centers}

In this data center network, each data center may fail, and its lifetime of staying in the network follows an exponential distribution with failure rate $\lambda$. On the other hand, new data centers continuously join to the data center network, and their inputs are a Poisson process with arrival rate $\beta$.

Let $N(t)$ be the number of available data centers normally operating in the data center network at the time $t$. Then $\{N(t): t \geq 0\}$ is a birth-death process on state space $\Omega=\{0,1,2, \ldots\}$ whose state transition relation is shown in Figure 1 .

From Figure 1, the infinitesimal generator of the birth and death process $\{N(t): t \geq 0\}$ is given by

$$
Q=\left(\begin{array}{cccc}
-\beta & \beta & & \\
\lambda-(\lambda+\beta) & \beta & & \\
2 \lambda & -(2 \lambda+\beta) & \beta & \\
& & \ddots & \ddots
\end{array}\right)
$$

Since the two numbers $\lambda$ and $\beta$ are fixed, there must exist a positive integer $n_{0}>\lfloor\beta / \lambda\rfloor+1$ such that when $n>n_{0}$, we have $\lambda n>\beta$, where $\lfloor x\rfloor$ is the maximal integer part of the real number $x$. Thus, by using the mean-draft condition, it is easy to see that the birth-death process $\{N(t): t \geq 0\}$ is irreducible, aperiodic and positive recurrent.

Let

$$
\begin{gathered}
\mathbf{N}=\lim _{t \rightarrow+\infty} N(t), \\
\theta_{k}=P\{\mathbf{N}=k\}, k=0,1,2, \ldots, \\
\theta=\left(\theta_{0}, \theta_{1}, \theta_{2}, \theta_{3}, \ldots\right) .
\end{gathered}
$$

Then it is clear that $\theta Q=0, \theta e=1$, where $e$ is a column vector with each element one.

Theorem 1 In this data centers network, the steady state number $\mathbf{N}$ of available data centers operating normally follows a Poisson distribution with parameter $\beta / \lambda$, that is

$$
\theta_{k}=\exp \left\{-\frac{\beta}{\lambda}\right\} \frac{1}{k !}\left(\frac{\beta}{\lambda}\right)^{k}, \quad k=0,1,2, \ldots
$$

Proof: By solving the linear equations $\theta Q=0, \theta e=1$, we get

$$
\theta_{k}=\exp \left\{-\frac{\beta}{\lambda}\right\} \frac{1}{k !}\left(\frac{\beta}{\lambda}\right)^{k}, \quad k=0,1,2, \ldots,
$$




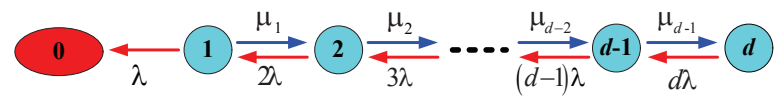

Fig. 2. State transition relation of a file replicated process

Thus the steady state number $\mathbf{N}$ of available data centers operating normally follows a Poisson distribution with parameter $\beta / \lambda$. This completes the proof. $\square$

It is seen from Theorem 1 that the Poisson random variable $\mathbf{N}$ provides useful information to understand the file $d$-replication policy. For example, the probability that no file can be successfully duplicated to a data center is given by $P\{\mathbf{N}=0\}=\exp \{-\beta / \lambda\}$

\subsection{An approximate assessment for file lifetime}

In this subsection, we first correct the replication rate of the files to the data center network by means of the steady state (Poisson) probability $\theta_{k}$ for $k \geq 0$. Then we establish a new finite-state birth-death process to provide an approximate assessment for the file lifetime.

By using Theorem 1 and

$$
\theta_{k}=\exp \left\{-\frac{\beta}{\lambda}\right\} \frac{1}{k !}\left(\frac{\beta}{\lambda}\right)^{k}, \quad k=0,1,2, \ldots,
$$

thus we can correct the replication rate of the files to the data centers as follows:

(a) If there is only a file in the data center network, then the file has the replication rate to another data center, given by

$$
\mu_{1}=\mu \sum_{j=2}^{\infty} \theta_{j}=\mu \sum_{j=2}^{\infty} \exp \left\{-\frac{\beta}{\lambda}\right\} \frac{1}{j !}\left(\frac{\beta}{\lambda}\right)^{j} .
$$

That is, the copying time of this file follows an exponential distribution with copy rate $\mu_{1}$.

(b) If there are $k$ identical copy files in the data center network for $2 \leq k \leq$ $d-1$, then the $k$ file has the replication rate to another data center, given by

$$
\mu_{k}=k \mu \sum_{j=k+1}^{\infty} \theta_{j}=k \mu \sum_{j=k+1}^{\infty} \exp \left\{-\frac{\beta}{\lambda}\right\} \frac{1}{j !}\left(\frac{\beta}{\lambda}\right)^{j} .
$$

That is, the copying time of the $k$ identical copy files follows an exponential distribution with copy rate $\mu_{k}$.

In the data center network, we denote by $M(t)$ the number of identical copy files of one file at the time $t$, then $\{M(t): t \geq 0\}$ is a birth-death process on a finite state space $\mathbf{E}=\{0,1,2, \ldots, d-1, d\}$ whose state transition relation is depcited in Figure 2. 
Let

$$
\eta=\inf \{t \geq 0: M(t)=0\},
$$

Then $\eta$ is the lifetime of a file which stays in the data center network. Of course, it is also the first lost time of the file which will possibly disappear in the data center network.

We write

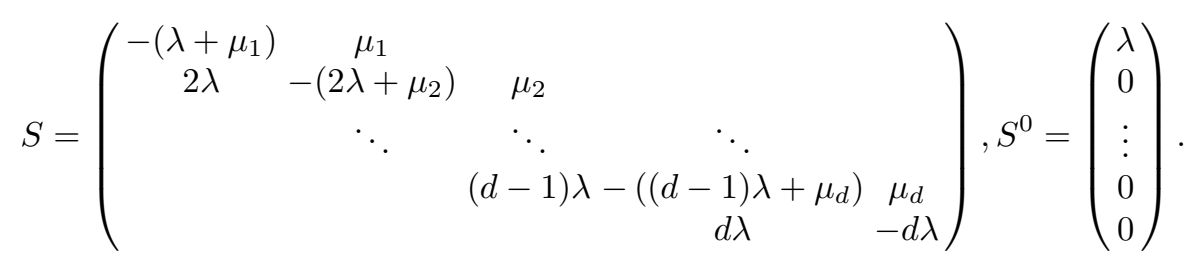

Also, we take the initial probability $\left(\widetilde{\gamma}, \gamma_{0}\right)$, where $\widetilde{\gamma}=\left(\gamma_{1}, \gamma_{2}, \ldots, \gamma_{d}\right), \gamma_{0} \in[0,1]$, and $\widetilde{\gamma} e=1-\gamma_{0}$.

Theorem 2 In this data center network, the lifetime $\eta$ of a file follows a PH distribution of size d with an irreducibility representation $(\widetilde{\gamma}, S)$. Also, the Markov process $\left(S+S^{0} \widetilde{\gamma}\right)$ is irreducible. Further, the kth moment of the lifetime $\eta$ of a file is given by

$$
E\left[\eta^{k}\right]=(-1)^{k} k ! \widetilde{\gamma} S^{-k} e, \quad k=1,2,3, \ldots
$$

Proof: It is easy to check that the infinitesimal generator of the birth-death process $\{M(t): t \geq 0\}$ on state space $\mathbf{E}=\{0,1,2, \ldots, d-1, d\}$ is given by

$$
\mathbf{Q}=\left(\begin{array}{cc}
S & S^{0} \\
0 & 0
\end{array}\right)
$$

Obviously, the lifetime $\eta$ of a file follows a PH distribution of size $d$ with an irreducibility representation $(\widetilde{\gamma}, S)$. Also, the Markov process $\left(S+S^{0} \widetilde{\gamma}\right)$ is irreducible. In addition, some simple computation can lead to the $k$ th moment of the lifetime $\eta$. This completes the proof.

Note that the matrix $S$ is the infinitesimal generator of a birth-death process, thus we can give expression for the inverse of matrix $S$. To this end, we write

$$
S^{-1}=\left(\begin{array}{ccccc}
S_{1,1} & S_{1,2} & \cdots & S_{1, d-1} & S_{1, d} \\
S_{2,1} & S_{2,2} & \cdots & S_{2, d-1} & S_{2, d} \\
\vdots & \vdots & & \vdots & \vdots \\
S_{d-1,1} & S_{d-1,2} & \cdots & S_{d-1, d-1} & S_{d-1, d} \\
S_{d, 1} & S_{d, 2} & \cdots & S_{d, d-1} & S_{d, d}
\end{array}\right)
$$

It is easy check from $S S^{-1}=I$ that the first column of $S^{-1}$ is given by

$$
s_{j, 1}=-\frac{1}{\lambda}, \quad 1 \leq j \leq d,
$$


and for $2 \leq k \leq d$, the $k$ th column of $S^{-1}$ is given by

$$
\begin{aligned}
s_{1, k} & =-\frac{\prod_{j=1}^{k-1} \mu_{j}}{k ! \lambda^{k}}, s_{2, k}=-\frac{\left(\lambda+\mu_{1}\right) \prod_{j=2}^{k-1} \mu_{j}}{k ! \lambda^{k}}, s_{3, k}=-\frac{2 ! \lambda^{2}+\left(\lambda+\mu_{1}\right) \prod_{j=3}^{k-1} \mu_{j}}{k ! \lambda^{k}}, \\
\ldots, s_{k-1, k} & =-\frac{\left[(k-2) ! \lambda^{k-2}+(k-3) ! \lambda^{k-3} \mu_{k-2}+\cdots+\lambda \prod_{j=2}^{k-2} \mu_{j}+\prod_{j=1}^{k-2} \mu_{j}\right] \mu_{k-1}}{k ! \lambda^{k}},
\end{aligned}
$$

and for $k \leq j \leq d$,

$$
s_{j, k}=-\frac{(k-1) ! \lambda^{k-1}+(k-2) ! \lambda^{k-2} \mu_{k-1}+\cdots+\lambda \prod_{j=2}^{k-1} \mu_{j}+\prod_{j=1}^{k-1} \mu_{j}}{k ! \lambda^{k}} .
$$

Thus we obtain

$$
E[\eta]=-\sum_{j=1}^{d} \sum_{i=1}^{d} \gamma_{i} s_{i, j}
$$

\section{A Two-Dimensional Assessment of File Lifetime}

In this section, we establish a two-dimensional Markov process by means of the number of available data centers and the number of identical copy files of one file. Based on this, we propose a two-dimensional assessment method of file lifetime in the data center network.

In the data center network, as seen above, let $N(t)$ and $M(t)$ be the numbers of available data centers and of identical copy files of one file at the time $t$, respectively. Obviously, $N(t) \in\{0,1,2, \ldots\}$ and $M(t) \in\{0,1,2, \ldots, d\}$. It is seen from the exponential and Poisson assumptions that $\{N(t), M(t): t \geq 0\}$ is a two-dimensional Markov process, and further a QBD process, whose state transition relation is depicted in Figure 3.

It is seen from Figure 3 that the state space of the QBD process $\{N(t), M(t): t \geq 0\}$ is expressed as

$$
\Theta=\Delta \cup \Theta_{1} \cup \Theta_{2} \cup \Theta_{3} \cup \cdots=\Delta \cup\left(\bigcup_{k=1}^{\infty} \Theta_{k}\right),
$$

where $\Delta=\{(k, 0): k=0,1,2 \ldots\}$ is a set of all the absorption states, which are written as an absorbing state $\boldsymbol{\Delta}^{*}$. Observing the columns in Figure 3, we write

Level $k \in\{1,2,3, \ldots, d-1\}: \Theta_{k}=\{(k, 1),(k, 2), \ldots,(k, k)\}$;

Level $l \in\{d, d+1, d+2, \ldots\}: \Theta_{l}=\{(l, 1),(l, 2), \ldots,(l, d)\}$. 


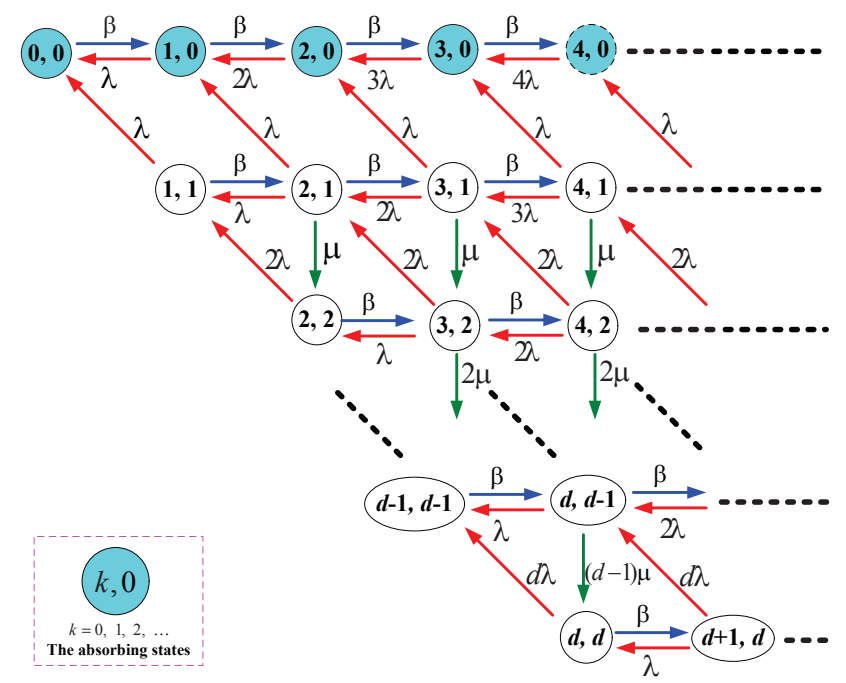

Fig. 3. State transition relation of a QBD process

From these levels, the infinitesimal generator of the QBD process $\{N(t), M(t): t \geq 0\}$ on sub-state space $\bigcup_{k=1}^{\infty} \Theta_{k}$ is given by

$$
T=\left(\begin{array}{cccccc}
A_{1,1} & A_{1,2} & & & \\
A_{2,1} & A_{2,2} & A_{2,3} & & \\
& A_{3,2} & A_{3,3} & A_{3,4} & \\
& & \ddots & \ddots & \ddots
\end{array}\right)
$$

where $\zeta_{j}(k)=k \lambda+\beta+j \mu$, and

$$
A_{1,1}=-(\lambda+\beta), A_{1,2}=(\beta, 0) ;
$$

for $2 \leq k \leq d$,

$$
\begin{aligned}
& A_{k, k-1}=\left(\begin{array}{ccccc}
(k-1) \lambda & & & & \\
2 \lambda & (k-2) \lambda & & & \\
& 3 \lambda & (k-3) \lambda & & \\
& & \ddots & \ddots & \\
& & & (k-1) \lambda & \lambda \\
& & & 0 & k \lambda
\end{array}\right), \\
& A_{k, k}=\left(\begin{array}{ccccc}
-\zeta_{1}(k) & \mu & & & \\
& -\zeta_{2}(k) & 2 \mu & & \\
& & \ddots & \ddots & \\
& & -\zeta_{k-1}(k) & (k-1) \mu \\
& & & & -\zeta_{0}(k)
\end{array}\right), \quad A_{k, k+1}=\left(\begin{array}{cccc}
\beta & & & \\
& \beta & & \\
& \beta & & \\
& & \beta & \\
& & \beta & 0
\end{array}\right) ;
\end{aligned}
$$


and for $l \geq d+1$,

$$
\begin{aligned}
& A_{l, l-1}=\left(\begin{array}{ccccc}
(l-1) \lambda & & & & \\
2 \lambda & (l-2) \lambda & & & \\
& 3 \lambda & (l-3) \lambda & & \\
& & \ddots & \ddots & \\
& & & (d-1) \lambda(l-d+1) \lambda \\
& & & d \lambda & (l-d) \lambda
\end{array}\right)
\end{aligned}
$$

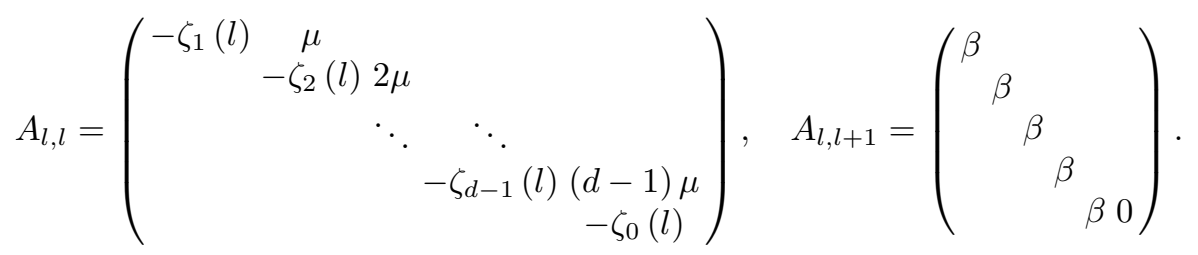

Further, the infinitesimal generator of the QBD process $\{N(t), M(t): t \geq 0\}$ on a modified state space $\boldsymbol{\Delta}^{*} \cup\left(\bigcup_{k=1}^{\infty} \Theta_{k}\right)$ is given by

$$
\mathbf{Q}=\left(\begin{array}{cc}
T^{0} & T \\
0 & 0
\end{array}\right)
$$

where

$$
T^{0}=-T e=(\lambda ; \lambda, 0 ; \lambda, 0,0 ; \lambda, 0,0,0 ; \lambda, 0,0,0,0 ; \ldots)^{T},
$$

and $a^{T}$ represents the transpose of the row vector $a$.

Let

$$
\chi=\inf \{t \geq 0: M(t)=0, N(t) \in\{0,1,2, \ldots\}\},
$$

Then the random variable $\chi$ is the first passage time that the QBD process $\{N(t), M(t): t \geq 0\}$ reaches the absorption state $\boldsymbol{\Delta}^{*}$ for the first time. That is, the random variable $\chi$ is the lifetime of a file of staying in data center network.

To use the PH distribution, we take an initial probability vector $\alpha=\left(\alpha_{\Delta^{*}}, \alpha_{1}, \alpha_{2}, \alpha_{3}, \ldots\right)$, and $\alpha_{\Delta^{*}} \in[0,1]$. For $1 \leq k \leq d$,

$$
\alpha_{k}=\left(\alpha_{k, 1}, \alpha_{k, 2}, \cdots, \alpha_{k, k-1}, \alpha_{k, k}\right)
$$

and for $l \geq d+1$,

$$
\alpha_{l}=\left(\alpha_{l, 1}, \alpha_{l, 2}, \cdots, \alpha_{l, d-1}, \alpha_{l, d}\right) .
$$

Theorem 3 In this data center network, the first passage time $\chi$ is an infinitedimensional $P H$ distribution with an irreducible representation $(\widetilde{\alpha}, T)$, where $\widetilde{\alpha}=$ $\left(\alpha_{1}, \alpha_{2}, \alpha_{3}, \cdots\right), \widetilde{\alpha} e=1-\alpha_{\Delta^{*}}$. Also, the Markov Process $T+T^{0} \widetilde{\alpha}$ is irreducible. Further, the kth moment of the first passage time $\chi$ is given by

$$
E\left[\chi^{k}\right]=(-1)^{k} k ! \widetilde{\alpha} T^{-k} e, \quad k=1,2,3, \ldots
$$


Proof: Corresponding to the modified state space $\boldsymbol{\Delta}^{*} \cup\left(\bigcup_{k=1}^{\infty} \Theta_{k}\right)$, the infinitesimal generator of the QBD process $\{N(t), M(t): t \geq 0\}$ is given by $\mathbf{Q}$. Thus it is clear that the first passage time $\chi$ is an infinite-dimensional PH distribution with an irreducible representation $(\widetilde{\alpha}, T)$. Also, the Markov Process $T+T^{0} \widetilde{\alpha}$ is irreducible, and the $k$ th moment of the first passage time $\chi$ is also obtained. This completes the proof.

It is necessary to show that the $R G$-factorizations by Li [8] can be applied to effectively deal with the infinite-dimensional $\mathrm{PH}$ distribution. Now, we calculate the mean $E[\chi]$. To this end, we first need to derive the inverse matrix of the matrix $T$ by using the $R G$-factorizations.

we define the $U$-measure as

$$
\mathbf{U}_{0}=A_{1,1},
$$

and for $k=1,2,3, \ldots$,

$$
\mathbf{U}_{k}=A_{k+1, k+1}+A_{k+1, k}\left(-U_{k-1}\right)^{-1} A_{k, k+1} .
$$

We respectively define the $R$ - and $G$-measures as

$$
\begin{aligned}
\mathbf{R}_{k} & =A_{k+1, k}\left(-\mathbf{U}_{k-1}\right)^{-1}, k=1,2,3, \ldots ; \\
\mathbf{G}_{l} & =\left(-\mathbf{U}_{l}\right)^{-1} A_{l+1, l+2}, \quad l=0,1,2, \ldots
\end{aligned}
$$

By using the Theorem 1 in Section 2.1 of Li and Cao [9], the $R G$-factorization of matrix $T$ is given by

$$
T=\left(I-\mathbf{R}_{L}\right) \mathbf{U}_{D}\left(I-\mathbf{G}_{U}\right),
$$

where

$$
\begin{aligned}
& \mathbf{U}_{D}=\operatorname{diag}\left(\mathbf{U}_{0}, \mathbf{U}_{1}, \mathbf{U}_{2}, \ldots\right),
\end{aligned}
$$

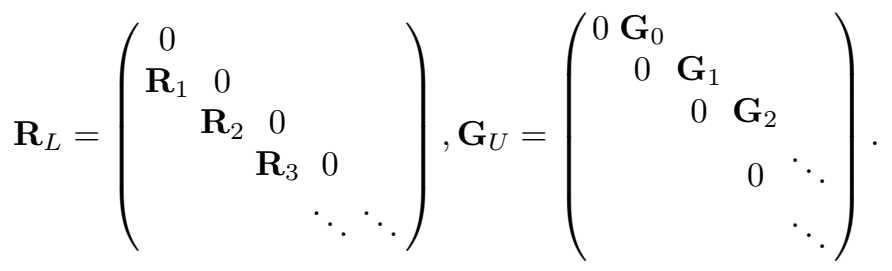

Thus we have obtain

$$
T^{-1}=\left(I-\mathbf{G}_{L}\right)^{-1} \mathbf{U}_{D}^{-1}\left(I-\mathbf{R}_{L}\right)^{-1} .
$$

Let

$$
\begin{aligned}
X_{k}^{(l)}=\mathbf{R}_{l} \mathbf{R}_{l-1} \cdots \mathbf{R}_{l-k+1}, & 1 \leq k \leq l, \\
Y_{k}^{(l)}=\mathbf{G}_{l} \mathbf{G}_{l+1} \cdots \mathbf{R}_{l+k-1}, & 0 \leq l \leq k,
\end{aligned}
$$

Then

$$
\mathbf{U}_{D}^{-1}=\operatorname{diag}\left(\mathbf{U}_{0}^{-1}, \mathbf{U}_{1}^{-1}, \mathbf{U}_{2}^{-1}, \ldots\right),
$$




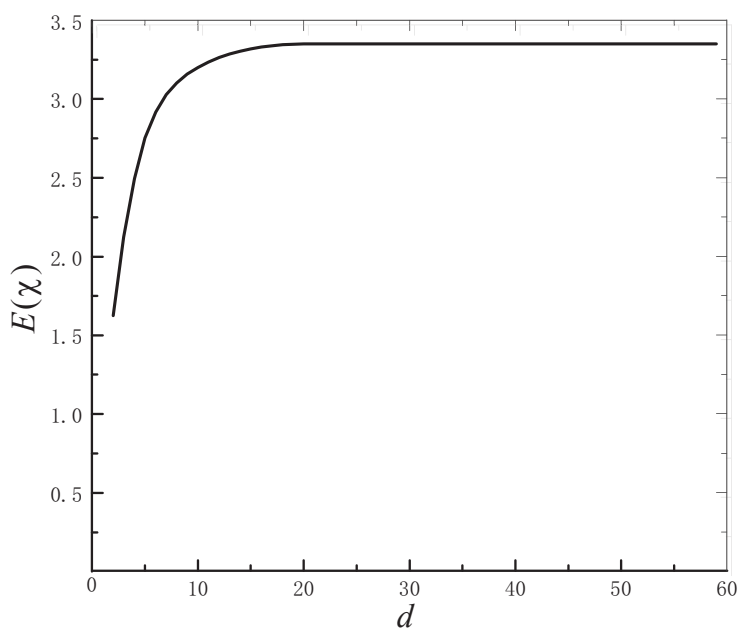

Fig. 4. The file lifetime depends on the key parameter $d$

and

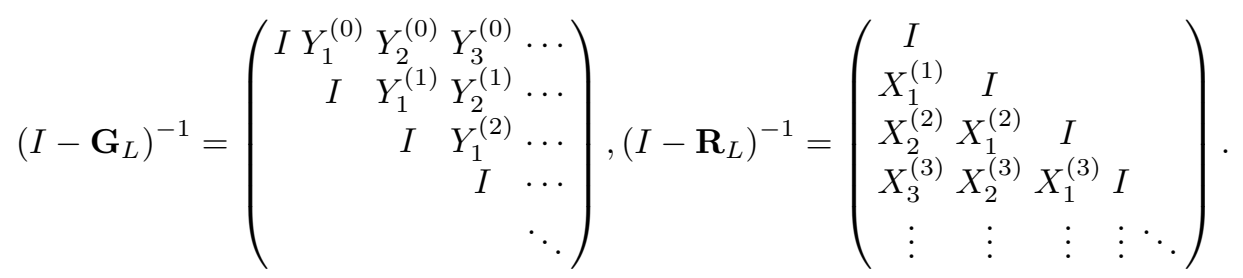

Let

$$
T^{-1}=\left(I-\mathbf{G}_{L}\right)^{-1} \mathbf{U}_{D}^{-1}\left(I-\mathbf{R}_{L}\right)^{-1}=\left(\begin{array}{cccc}
T_{0,0} & T_{0,1} & T_{0,2} & \cdots \\
T_{1,0} & T_{11} & T_{1,2} & \cdots \\
T_{2,0} & T_{2,1} & T_{2,2} & \cdots \\
\vdots & \vdots & \vdots & \ddots
\end{array}\right) .
$$

Then the mean of the first passage time $\chi$ is given by

$$
E[\chi]=-\sum_{j=0}^{\infty} \sum_{i=0}^{\infty} \alpha_{i+1} T_{i, j} e .
$$

Finally, we use a simple example to illustrate how the file lifetime $\chi$ depends on the maximum number of identical backups: $d \in(2,59)$. Let $\lambda=1$ and $\beta=4$. As seen form figure 4 , the mean $E[\chi]$ increases, as $d$ increases. In addition, when $d$ increases to a certain value, the mean $E[\chi]$ will no longer change significantly. Such a phenomenon will be very useful in design and optimization of the data center network with file replication mechanism. 


\section{References}

1. Aghajani, R., Robert, P., Sun, W.: A large scale analysis of unreliable stochastic networks. The Annals of Applied Probability 28(2), 851-887 (2018)

2. Blake, C., Rodrigues, R.: High availability, scalable storage, dynamic peer networks: Pick two. In: The 9th Workshop on Hot Topics in Operating Systems, Vol. 3, pp. 18-21. (2003)

3. Chun, B.G., Dabek, F., Haeberlen, A. et al.: Efficient replica maintenance for distributed storage systems. In: The 3rd Symposium on Networked Systems Design \& Implementation, Vol. 6, pp. 45-58. (2006)

4. Feuillet, M., Robert, P.: A scaling analysis of a transient stochastic network. Advances in Applied Probability 46(2), 516-535 (2014)

5. Kersch, P., Szabo, R.: Mathematical modeling of routing in DHTs. In: Handbook of Peer-to-Peer Networking, pp. 367-401. Springer, Boston (2010)

6. Kniesburges, S., Koutsopoulos, A., Scheideler, C.: CONE-DHT: A Distributed selfstabilizing algorithm for a heterogeneous storage system. In: International Symposium on Distributed Computing, pp. 537-549. Springer, Berlin, Heidelberg (2013)

7. Lian, Q., Chen, W., Zhang, Z.: On the impact of replica placement to the reliability of distributed brick storage systems. In: The 25th IEEE International Conference on Distributed Computing Systems, pp. 187-196. IEEE (2005)

8. Li, Q.L.: Constructive computation in stochastic models with applications: the RGfactorizations. Springer (2010)

9. Li, Q.L., Cao, J.: Two types of RG-factorizations of quasi-birth-and-death processes and their applications to stochastic integral functionals. Stochastic models 20(3), 299-340 (2004)

10. Pace, A., Quema, V., Schiavoni, V.: Exploiting node connection regularity for DHT replication. In: The 30th IEEE Symposium on Reliable Distributed Systems, pp. 111-120. IEEE (2011)

11. Picconi, F., Baynat, B., Sens, P.: An analytical estimation of durability in DHTs. In: International Conference on Distributed Computing and Internet Technology, pp. 184-196. Springer, Berlin, Heidelberg (2007)

12. Picconi, F., Baynat, B., Sens, P.: Predicting durability in dhts using Markov chains. In: The 2nd International Conference on Digital Information Management, Vol. 2, pp. 532-538. IEEE (2007)

13. Pinheiro, E., Weber, W.D., Barroso, L.A.: Failure trends in a large disk drive population. In: The 5th USENIX Conference on File and Storage Technologies, Vol. 7, No. 1, pp. 17-23. (2007)

14. Ramabhadran, S., Pasquale, J.: Analysis of long-running replicated systems. In: INFOCOM, Vol. 2006, pp. 1-9. (2006)

15. Ramabhadran, S., Pasquale, J.: Durability of replicated distributed storage systems. ACM SIGMETRICS Performance Evaluation Review 36(1), 447-448 (2008)

16. Ramabhadran, S., Pasquale, J.: Analysis of durability in replicated distributed storage systems. In: IEEE International Symposium on Parallel \& Distributed Processing, pp. 1-12. IEEE (2010)

17. Sun, W., Feuillet, M., Robert, P.: Analysis of large unreliable stochastic networks. The Annals of Applied Probability 26(5), 2959-3000 (2016)

18. Utard, G., Vernois, A.: Data durability in peer to peer storage systems. In: IEEE International Symposium on CLUSTER Computing and the Grid, 90-97. IEEE (2004) 